\section{Further evidence for the spread of attention during contour grouping: A reply to Crundall, Dewhurst, and Underwood (2008)}

\author{
Pieter R. Roelfsema \\ Netherlands Institute for Neuroscience, \\ Amsterdam, The Netherlands
}

and Vrije Universiteit Amsterdam, Amsterdam, The Netherlands

RoOs HouTKAMP

Netherlands Institute for Neuroscience, Amsterdam, The Netherlands

and Otto-von-Guericke Universität, Magdeburg, Germany

AND

ILIA KORJOUKOV

Netherlands Institute for Neuroscience, Amsterdam, The Netherlands

In a contour-grouping task, subjects decide whether contour elements belong to the same or different curves. Houtkamp, Spekreijse, and Roelfsema (2003) demonstrated that objectbased attention spreads gradually over contour elements that have to be grouped in perception. Crundall, Dewhurst, and Underwood (2008) challenged this spreading-attention model and suggested that attention in the contour-grouping task is not object based but rather has the shape of a zoom lens that moves along the relevant curve. To distinguish between object-based and spatial attention, they changed the stimulus and measured the impact on performance. Subjects were not able to correct for changes at the start of the relevant curve toward the end of the trial. They suggested that attention did not stay at the beginning of the curve, in accordance with a moving zoom lens model. Here, we examine the task of Crundall et al. and find that subjects perceive the changes but fail to correct their response. By measuring change detection directly, we find that performance is much better for the start of the relevant curve than for an irrelevant curve, at all times. Our findings do not support the zoom lens model but provide further support for the spreadingattention model.

The typical visual scene that we perceive is cluttered with many objects embedded in a complex background. To analyze this wealth of information, our visual system starts with a decomposition of the image into small, digestible parts. The first steps of image processing are carried out by neurons with small receptive fields that oversee only a small fraction of the incoming information and that are tuned to simple features, such as line elements of a particular orientation and surface patches with a specific color or texture. This piecemeal analysis is very different from our subjective perception. We do not perceive a set of small image fragments but interact with spatially extended objects that may fill hundreds or thousands of receptive fields. Thus, our visual system must be equipped with powerful perceptual-grouping processes that reconstruct objects from these image fragments. We appear to perceive the objects immediately, even if they are large, and it is easy for us to tell where in the picture one object ends and the next one begins.

Neurons in higher areas of the visual cortex presumably account for some of this efficiency. These neurons have large receptive fields and are tuned to complex objects, like faces and other shapes (Oram \& Perrett, 1994; Riesenhuber \& Poggio, 1999; Roelfsema, 2006; Tanaka, 1993; Tsao, Freiwald, Tootell, \& Livingstone, 2006), and they are activated only a few tens of milliseconds after the neurons in early visual areas (Hung, Kreiman, Poggio, \& DiCarlo, 2005; Oram \& Perrett, 1992; Sugase, Yamane, Ueno, \& Kawano, 1999). The activity of a neuron tuned to, say, a face may explain how the visual brain rapidly detects the group of image elements that belong to such a familiar object (Rousselet, Macé, \& Fabre-Thorpe, 2003; Thorpe, Fize, \& Marlot, 1996). The efficient detection of groups of image elements by selective neurons in higher areas of the visual cortex has been called base grouping (Roelfsema, 2006).

However, there are also perceptual-grouping tasks that demand more processing time. An example of such a task is shown in Figure 1A. Imagine approaching the desk because you want to switch on the light. To determine which plug to put into the socket, you have to know which plug is attached to the lamp. This task could be solved by a perceptual-grouping operation that groups together all the contour elements of the lamp cable and segregates them from the contour elements of the other cable. In an elegant series of studies, Jolicœur and colleagues (Jolicœur \& Ingleton, 1991; Jolicœur, Ullman, \& Mackay, 1986, 1991) demonstrated that a laboratory version of this task requires many hundreds of milliseconds, and that the response time (RT) of subjects increases linearly with the number of contour elements that need to be grouped in perception. Later studies demonstrated that this grouping process also exhibits substantial delays if the relevant curve crosses with another curve, as is the case in Figure 1A. Every intersection between the relevant curve and another curve adds approximately

P. R. Roelfsema, p.roelfsema@nin.knaw.nl 
A

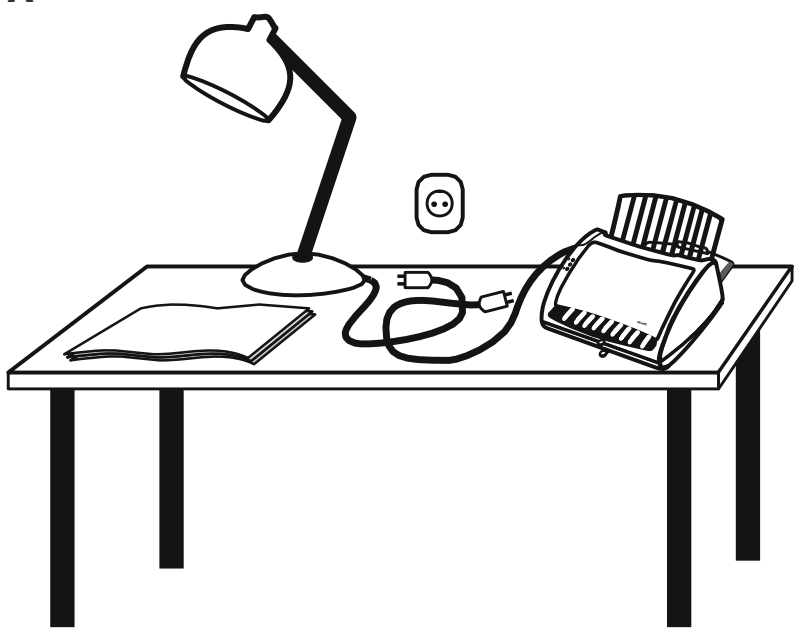

B

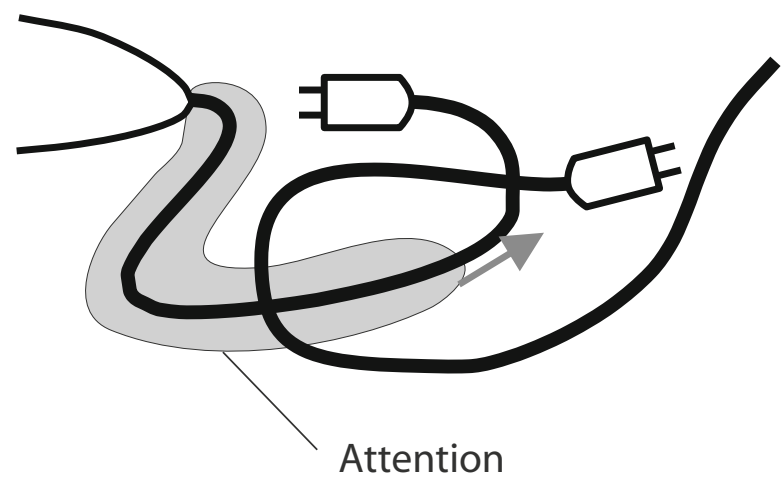

C

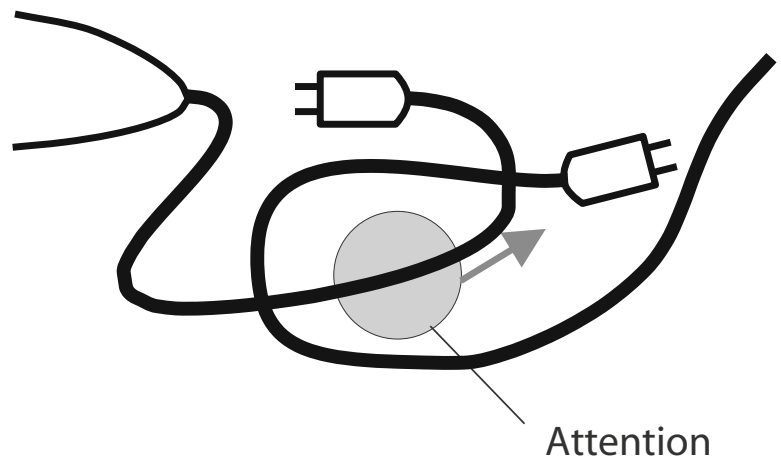

Figure 1. Attentional grouping of contour elements into elongated curves. (A) Curve-tracing task where you will have to group contour elements into an elongated cable if you want to switch on the light. (B) Attentional spreading model that assumes that attention gradually spreads until all contour elements of one of the cables are labeled by attention. (C) Moving zoom lens model that holds that a zoom lens moves along the cable to enhance the representation of only a small region at a time.
100 msec to the overall RT (Crundall, Dewhurst, \& Underwood, 2008; Houtkamp, Spekreijse, \& Roelfsema, 2003; Roelfsema, Scholte, \& Spekreijse, 1999; Scholte, Spekreijse, \& Roelfsema, 2001).

Why is this simple task solved with less efficiency than is the grouping of image elements belonging to a complex, familiar object? There may be at least two reasons. First, in the example of Figure 1A, there are no global features that distinguish the contour elements of one of the cables from the elements of the other cable. In this situation, the grouping of contour elements has to rely on local grouping cues. Nearby elements of the same curve are related to each other by the Gestalt grouping cues of connectedness and good continuation; that is, they are locally collinear and connected to each other. However, contour elements of the same curve that are farther apart do not have such a special relationship, and are only grouped indirectly, through a chain of local groupings. Second, the cables can be in many different configurations, and it is presumably impossible to reserve a single neuron in higher visual areas for every possible contour configuration. In these situations, perceptual grouping may have to occur at the representational level where the individual contour elements are coded, but this requires a mechanism for the labeling of neurons that code all the contour elements belonging to the same curve, so that they can be distinguished from neurons coding elements of different curves (Roelfsema, 2006). In neurophysiological experiments with configurations similar to the one in Figure $1 \mathrm{~A}$, we have observed that neurons coding the contour elements of a relevant curve are indeed labeled in the visual cortex, because they increase their activity over the activity of neurons coding irrelevant curves (Roelfsema, Lamme, \& Spekreijse, 1998). In recent experiments, we observed that the increase in neuronal activity starts at the beginning of the relevant curve and gradually spreads until all its elements are labeled with the enhanced response (Pooresmaeili \& Roelfsema, 2010). We have called this serial process of grouping by labeling with an enhanced response "incremental grouping" (Roelfsema, 2006).

Comparable increases of neuronal activity are observed frequently in neurophysiological studies in tasks requiring attention shifts (Reynolds \& Chelazzi, 2004). We therefore conjectured that attention might spread gradually from the initial segments of a target curve until the entire curve has been labeled with attention (Figure 1B; Houtkamp et al., 2003). Such a gradual spread would account for the finding that the RT of subjects increases linearly with the number of contour elements that need to be grouped together. Moreover, this view is consistent with studies showing that attention can be object based, so that it can be directed to the various parts of one object even if it overlaps with a distractor (Duncan, 1984). In addition, it is in accordance with the hypothesis that attention can select a "grouped array" of image elements at early representational levels (Vecera, 1994).

Spreading-attention models have also been proposed to explain the effects of Gestalt grouping in cuing and flanker tasks. In a seminal cuing study, Egly, Driver, and 
Rafal (1994) presented two rectangles and asked subjects to respond to the appearance of a target in one of these rectangles. The target was preceded by a valid or invalid cue; as expected, responses to targets presented at validly cued locations were faster. The remarkable finding was a benefit in RTs when the target was presented at a noncued location at the other side of the cued rectangle, compared with when it appeared on the noncued rectangle. This suggests that the cue attracted attention, which then spread across the entire rectangle (Avrahami, 1999; Egly et al., 1994). Lamy and Egeth (2002) demonstrated that this spread of attention within an object is not mandatory, however, and that object-based effects are particularly pronounced if the task demands an attention shift between locations. This attention shift is more efficient if these locations belong to the same object. Other studies that obtained evidence for the spread of attention used the Eriksen flanker task (Eriksen \& Eriksen, 1974). In this task, subjects report the features of a central item flanked by response-compatible or response-incompatible items. The usual finding is that the RT is shorter if the target is accompanied by response-compatible flankers than if it is accompanied by incompatible ones, as if subjects were not able to focus attention on the central target and thereby exclude the flankers. Flankers that form a perceptual group with the target because they are connected to it (Kramer \& Jacobson, 1991; Richard, Lee, \& Vecera, 2008), or in good continuation (Baylis \& Driver, 1992), increase the magnitude of the flanker effect. This result suggests that it is even more difficult to focus attention in the case of grouping, in accordance with the view that it would automatically spread along the groupings to the flankers. This automatic spreading-attention model was called into question by Shomstein and Yantis (2002), however, who demonstrated that there are also conditions under which perceptual grouping has little effect in the flanker task. Later studies examined the conditions where the effects of grouping in the flanker do and do not occur. Robust effects are observed when the task-relevant features and flanking features are integral parts of the objects (Richard et al., 2008), and also when the perceptual organization into grouped and ungrouped image parts is increased by other means (Chen \& Cave, 2006). Taken together, the previous results lend strong support to theories proposing that attention spreads according to the Gestalt grouping cues. In the curve-tracing task, the spread of attention eventually highlights all image elements of a traced curve, and it can thereby group them into a coherent representation.

However, the spreading-attention, object-based model for contour grouping is not the only model that can explain serial processing. Another model forwarded by Jolicœur et al. (1991; McCormick \& Jolicœur, 1994) holds that the task is solved by a zoom lens of attention that starts at the beginning of the relevant curve and then moves along the curve (Figure 1C). The task of Figure 1A is solved when the zoom lens reaches the correct plug, but grouping of the whole curve does not occur in this model, because attention does not label all contour elements simultaneously. The major difference between the spreading-attention and zoom lens models is in the maintenance of attention on the beginning of the curve. The spreading-attention model predicts that attention stays on these contour elements and the zoom lens model predicts that it does not.

To distinguish between these models, Houtkamp et al. (2003) combined a primary curve-tracing task with a secondary color-report task. The primary task had been introduced by Roelfsema et al. (1999) and is illustrated in Figure 2. Subjects saw two curves that could cross each other and indicated whether the target curve ( $\mathrm{T}$ in Figure $2 \mathrm{~A}$ ), starting at the fixation point, was connected to a larger circle at the left or right bottom of the stimulus. The stimuli differed at three locations that will be called "critical zones." At the first critical zone, the fixation point was connected to either the left or the right curve, and at the other critical zones, the two curves intersected with each other or stayed separate, giving rise to a total of eight stimuli (four of these are shown in Figure 2A; the other four were vertical mirror images). Here, we will refer to these stimuli as $\mathrm{OO}, \mathrm{OX}, \mathrm{XO}$, and $\mathrm{XX}$, where an $\mathrm{O}$ denotes a nonintersection and $\mathrm{X}$ an intersection (using the notation of Crundall et al., 2008). The main finding of Roelfsema et al. (1999) was that every intersection adds approximately $100 \mathrm{msec}$ to the RT, as if intersections reduced the efficiency of the contour-grouping process. To measure the distribution of attention during this task, Houtkamp et al. briefly presented colors on some of the contour elements (white segments in Figure 2B), asking the subjects to report the color of these contour elements after they responded in the contour-grouping task. Subjects were better in reporting the colors of the target curve than in reporting those of the distractor curve (D in Figure $2 \mathrm{~A}$ ), indicating that attention was directed to the target curve (see also Scholte et al., 2001). If the colors were shown at the start of the curve-tracing task, subjects were better at reporting colors of the target curve, but only for the contour elements at the beginning of this curve, close to the fixation point (locations 1t vs. 1d in Figure 2B). If the colors were shown later, performance was higher for all parts of the target curve (i.e., performance for $1 \mathrm{t}, 2 \mathrm{t}$, and $3 \mathrm{t}$ was better than that for $1 \mathrm{~d}, 2 \mathrm{~d}$, and $3 \mathrm{~d}$ ). The result that distinguishes between the models is the secondary task performance at location $1 \mathrm{t}$, which should stay high, according to the spreading-attention model, but should go down in the zoom lens model. Houtkamp et al. found that performance stays high for these contour elements, in accordance with the spreading-attention model.

Recently, Crundall et al. (2008) criticized Houtkamp et al. (2003) on two grounds. The first issue raised by Crundall et al. was the use of a secondary task, which may have had an influence on the subjects' strategy and also on the distribution of attention in the contourgrouping task. This is a valid concern that applies to all studies using a secondary task to infer the distribution of attention (we demonstrate below that it also applies to Crundall et al., 2008). However, Houtkamp et al. made an effort to reduce the likelihood of these strategy changes. They started the experiment with a baseline condition to measure performance in the absence of the secondary 


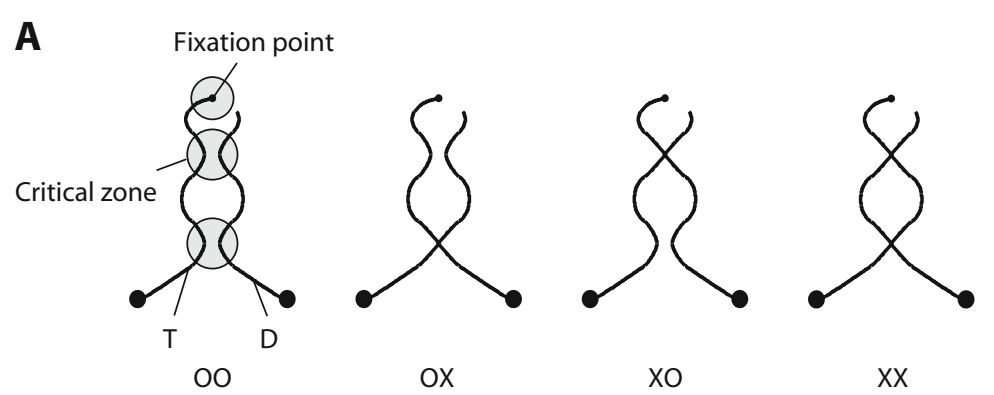

B

C
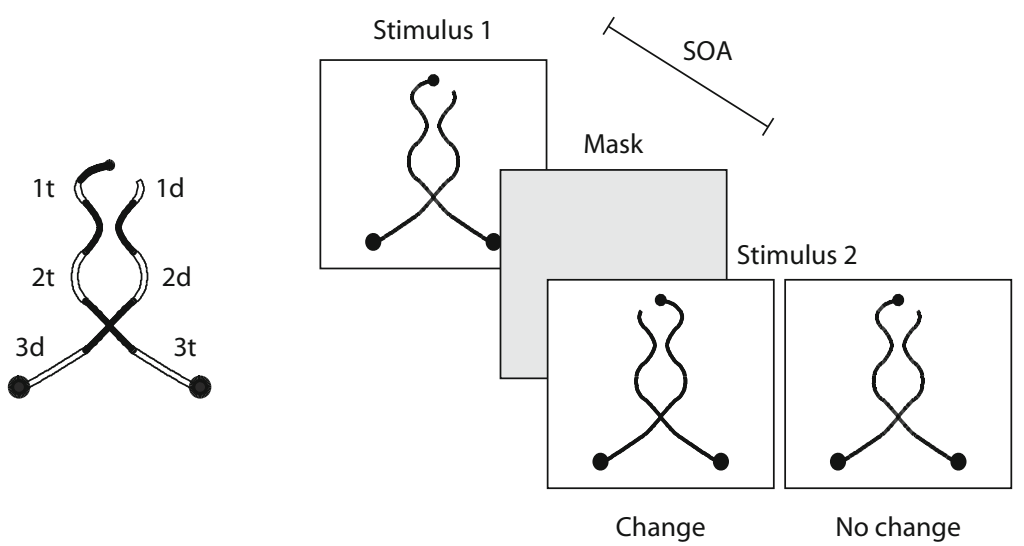

Figure 2. Previous experiments addressing the mechanisms for contour grouping. (A) Subjects judged whether the fixation point is connected to the left or right circle at the bottom of the stimulus by a target curve (T). The other curve is a distractor (D). On every trial the subject sees one of the stimuli or its mirror image (left/right inversion). $\mathrm{OO}, \mathrm{OX}, \mathrm{XO}$, and $\mathrm{XX}$ refer to the different stimulus categories, where $\mathrm{X}$ denotes an intersection and $O$ a nonintersection. (B) In the experiments of Scholte, Spekreijse, and Roelfsema (2001) and Houtkamp, Spekreijse, and Roelfsema (2003), contour elements were colored and subjects had a secondary task to report one of these colors (at locations 1t, 2t, 3t, 1d, 2d, or 3d) at the end of the trial. (C) In the study of Crundall, Dewhurst, and Underwood (2008) the stimulus changed on $50 \%$ of trials, and on these change trials the other circle became connected to the fixation point so that subjects had to reverse their response.

task, then switched to the dual task while emphasizing the importance of the primary contour-grouping task and urging the subjects to speed up if their RTs were longer than those in the single-task condition. This design was successful because curve-tracing performance in the dual task was not significantly different from when it was performed in isolation.

The second criticism raised by Crundall et al. (2008) is that the difference in performance between the target and distractor curve might not be caused by an attention shift, but rather by an effect of figure-ground assignment. Wong and Weisstein (1983) showed that the detection of high spatial frequencies is better in figural regions than in background regions in an ambiguous display where either a vase or two faces could be perceived as foreground. Crundall et al. suggested that the distractor curve in Figure 2A might have been perceived as background, and that this might have impaired the perception of its colors; we note, however, that there is little ambiguity in the stimulus about figure and background. In Figure 2A, we perceive two curves against a homogeneous background, and figureground reversal takes quite some effort (it would produce two curvy slits carved out of a white foreground surface); it is, therefore, unlikely that the effects observed by Houtkamp et al. (2003) are caused by figure-ground reversals. Interestingly, by increasing performance for higher spatial frequencies and decreasing it for lower spatial frequencies (Yeshurun \& Carrasco, 1998), selective attention also has an effect on the perception of spatial frequencies. We note, however, that even if the improved performance in the secondary task observed by Houtkamp et al. were caused, in part, by an attentional effect that increases the sensitivity to high spatial frequencies, the results would support the spreading-attention model, because the performance for the initial segment of the target curve (1t in Figure 2B) remained high until the end of the trial.

Crundall et al. (2008) took another approach to measure the distribution of attention during contour grouping. 
They combined the contour-grouping task of Roelfsema et al. (1999) with a change-detection task (Figure 2C). Changes in the stimulus occurred on $50 \%$ of trials, and they required the subjects to reverse their response in the contour-grouping task. The changes in the stimulus were difficult to detect, because they were masked with a global luminance transient ("mask" in Figure 2C). Change detection requires attention, and subjects should notice the change only if they attend to the changed location and should make an error if attention is directed elsewhere. The authors therefore used the subjects' performance on change trials as a measure for the distribution of attention in the contour-grouping task. A feature of this new method that seems desirable is that it may permit the measurement of attention without the need to use a secondary task.

Crundall et al. (2008) varied the location of the change in the stimulus across experiments. The change could occur either at the top of the stimulus (upper critical zone in Figure 2A) or at one of the two lower critical zones. Moreover, Crundall et al. varied the time of the change (i.e., the time between the initial stimulus and mask), to investigate the distribution of attention at the beginning of the curve-tracing task and at later points in time. The central finding was that subjects were more likely to make an error if the change in the stimulus occurred at a late time during the trial than if it occurred at the beginning, even if this change occurred in the first critical zone. In Crundall et al.'s Experiment 1, for example, the change occurred in the upper critical zone at a short stimulus onset asynchrony (SOA; the time between the first stimulus and the mask) of about $70 \mathrm{msec}$ (averaged across subjects), and the accuracy of the subjects decreased from about $85 \%$ on no-change trials to $75 \%$ on change trials. At a longer SOA of, on average, $400 \mathrm{msec}$, accuracy decreased more, from about $85 \%$ on no-change trials to $60 \%$ on change trials. The authors suggested that this finding provides evidence against the spreading-attention model, because the subjects should have noticed the change if their attention had stayed on the initial elements of the target curve, as predicted by the spreading-attention model. An attentional zoom lens, however, would have moved to a position farther along the curve, so that the change went unnoticed and the subjects made an error.

Unfortunately, a critical evaluation of the paradigm of Crundall et al. (2008) undermines these conclusions, for a number of reasons. First, we can predict the performance of subjects who do not perceive the change because it reverses the required response. If subjects do not perceive the change because the zoom lens has passed the location of the change, a performance of $85 \%$ should decrease to $15 \%$. In Crundall et al.'s Experiment 1, for example, the worst performance on change trials was $60 \%$, indicating that the subjects actually detected most changes even if these changes occurred at the start of the target curve and at the longer SOA. If change detection is impossible without attention, then we are forced to conclude that attention was still on the start of the target curve; this does not support the moving spotlight model. Moreover, it is not clear how the subjects handled the change trials. It is unknown whether detected changes require a new tracing process that might start either at the fixation point or at the location of the change. This leaves open the possibility that subjects detected the change but did not reverse their response in the curve-tracing task.

Second, it is very likely that any task manipulation that reverses the required response on $50 \%$ of the trials induces changes in the subjects' strategy. The method assumes that the location of the spotlight or the spread of attention is "frozen" during the abrupt visual transients caused by the homogeneous display used to mask the changes, and that the tracing process continues thereafter in an undisturbed manner. It is unlikely that this assumption holds, because some subjects may not even have considered the stimulus before the mask, since the premask stimulus was uncorrelated with the correct response. Other subjects may have retraced the stimulus after the mask, at least on a fraction of the trials. We can gain some insight into these strategy changes by evaluating the RT on the no-change trials. If the subjects simply continued tracing at the point where it was interrupted by the flicker, RT on no-change trials should not depend on the SOA. However, if the subjects ignored the stimulus before the flicker or if they retraced after the flicker, RT should increase with SOA. In all of Crundall et al.'s experiments, the RT on no-change trials depended strongly on SOA. In their Experiment 1 with a short SOA of $70 \mathrm{msec}$ and a long SOA of $400 \mathrm{msec}$, for example, the RT on no-change trials at the longer SOA increased by $200 \mathrm{msec}$ over that at the shorter SOA. This result implies that the experimental manipulation introduced to measure attention disturbed the very process it set out to evaluate.

Third, both the spreading-attention and the zoom lens models predict that changes at a location not yet reached by attention are not registered and do not influence performance. In contrast, Crundall et al. (2008) observed that changes at the lower critical zone degraded performance even at short and intermediate SOAs, and failed to observe an interaction between the timing and the location of the change predicted by both models. These criticisms, taken together, undermine Crundall et al.'s claims.

To test the validity of these criticisms, we will investigate Crundall et al.'s (2008) paradigm, but with a few modifications of the design. After each trial, we will ask the subjects whether or not they observed a change in the stimulus. This will allow us to distinguish between changes not perceived by the subjects and changes that were perceived but did not cause a change in their responses. Furthermore, we wish to establish the baseline change-detection performance for unattended material. As noted above, Crundall et al. observed that changes at the start of the relevant curve at a later point during a trial decreased performance from $85 \%$ to $60 \%$. Is this accuracy compatible with the withdrawal of attention? If so, we would expect a change-detection performance of $\sim 60 \%$ for unattended material. We will therefore add a second pair of irrelevant curves to the stimulus and compare change-detection performance between the relevant and irrelevant pairs of curves. 


\section{METHOD}

Five subjects with normal or corrected-to-normal vision participated in the experiment. They were naive about the purpose of the experiment. The experiment was subdivided into six sessions spread out over 3 or 4 days. The subjects sat at a distance of $53 \mathrm{~cm}$ from a 19-in. computer monitor. The setup was equipped with a chinrest and an EyeLink eyetracking device. Each trial began with a fixation dot and a short time interval $(300 \mathrm{msec})$ during which the subjects could monitor their own eye positions and recenter the eyetracking device, if necessary, by pressing the space button. Then the eye position was hidden, and each subject was requested to keep fixation inside a fixation window with a radius of $1.75^{\circ}$ centered on the fixation point for $700 \mathrm{msec}$ to start the trial. The first block was a training block with 128 trials, in which subjects practiced maintaining fixation on the fixation point during the curve-tracing task.

\section{Curve-Tracing Task-Baseline Trials}

In the second block of trials, subjects carried out 128 trials in a baseline task in which we measured the RT for the various types of stimuli. Every trial started with a fixation epoch of $1,000 \mathrm{msec}$ (as described in the previous paragraph), and then the stimulus appeared. It consisted of two pairs of white curves shown on a black background. One pair of curves was presented in the left lower quadrant and the other pair in the right lower quadrant of the visual field (Figure 3), and every pair of curves was chosen from the basic stimulus set shown in Figure 2A (OO, OX, XO, and XX). Only one curve (the target curve) was connected to the fixation point, and the subjects reported whether the item at the end of this curve was a blue diamond or a red circle by pressing one of two buttons on a gamepad device. The eccentricity of the item at the end of the target curve was approximately $13^{\circ}$. We will refer to the target curve and the adjacent distractor curve as the relevant pair, and to the other pair as irrelevant. There were eight possible configurations per pair: 4 types of crossing configurations $(\mathrm{OO}, \mathrm{OX}, \mathrm{XO}$, and $\mathrm{XX}) \times 2$ positions of the top line element (see circle labeled "close" in Figure 3) that determined which curve of the pair was the target curve. Finally, the fixation point was either connected to the left or the right pair, giving rise to a total of 128 stimuli $(2 \times 8 \times 8)$. One curve of each pair was attached to a red circle and the other one to a blue diamond (with a size of $0.8^{\circ}$ ). The stimulus stayed in view until the subject made a response or $5 \mathrm{sec}$ had passed. There were no changes in the stimulus during the baseline session. If the subject's eye position deviated from the fixation point by more than $1.75^{\circ}$ before the response, the trial was aborted and repeated later within the same block ( $18 \%$ of trials).

\section{Main Curve-Tracing Task, Including Change Trials}

Subjects carried out a total of four sessions of 128 trials each in a task that included change trials ( $50 \%$ of all the trials) in which the stimulus changed at one of four locations. These potential changes were masked by a gray screen that was shown for $100 \mathrm{msec}$. The trial started with the fixation point for $1,000 \mathrm{msec}$. Then the stimulus appeared; it was always followed by a mask and a second stimulus, which was either identical or changed. On change trials, we switched the connections either at the top critical zone of one of the pairs (close change) or at the lower critical zone (distal change): Here, an intersection could change into a nonintersection, or vice versa (dashed circles in Figure 3). All four possible changes occurred with equal probability, and they could occur after a short $(25 \%$ of all trials) or a long ( $25 \%$ of trials) SOA relative to the initial appearance of the stimulus (the other $50 \%$ were no-change trials). Note that a change in the relevant pair inverted the correct response in the tracing task, but that a change in the irrelevant pair did not require a

Fixation $(1,000 \mathrm{msec})$

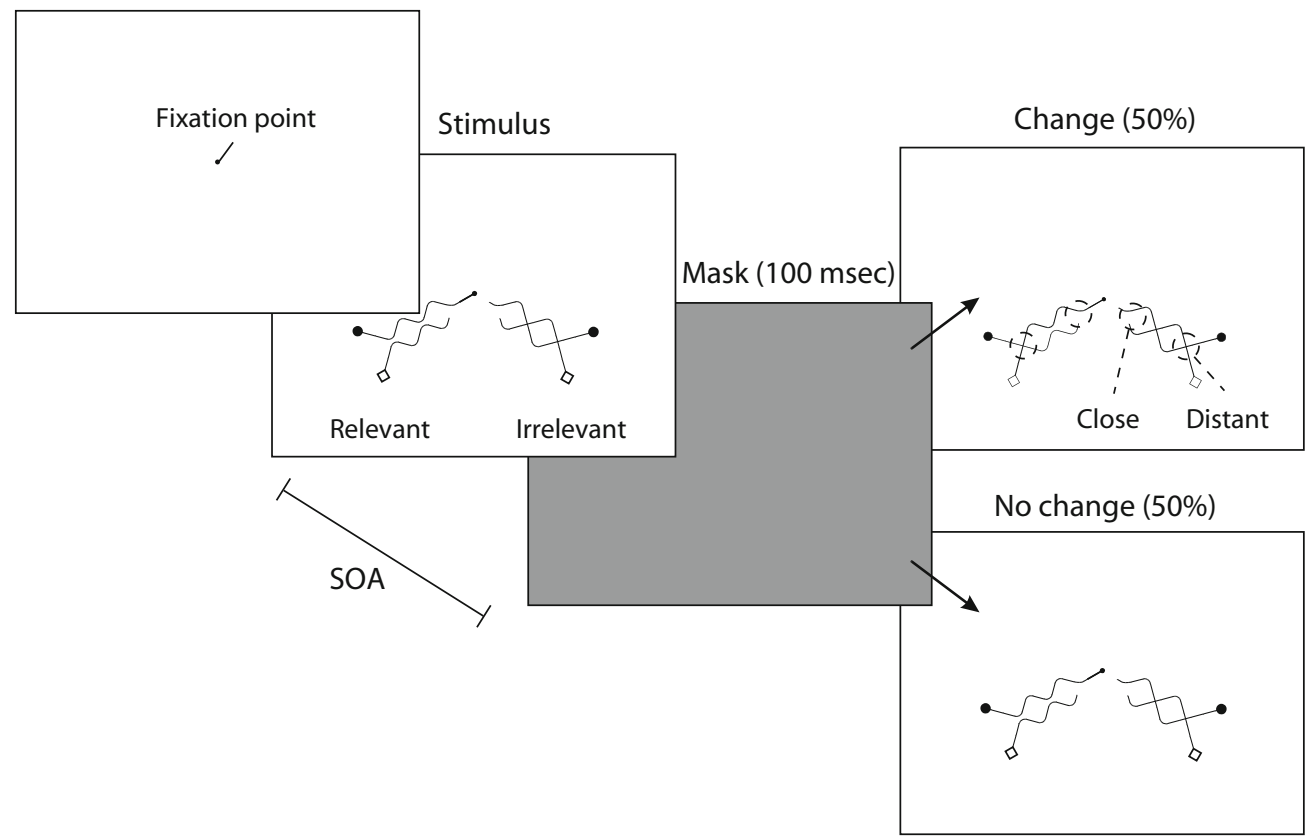

Figure 3. Contour-grouping task used in the present study. Subjects saw two pairs of curves, and only one of the curves (the target curve) was connected to the fixation point. Subjects judged whether the item at the other end of this curve was a red circle or a blue diamond. The pair of curves of which one was connected to the fixation point is called relevant, and the other pair is called irrelevant. On $50 \%$ of the trials, the contours changed at one of four possible locations that are indicated by dashed circles. At the less eccentric change locations ("close locations"), the upper curve segments were switched within a pair, and at the more eccentric change locations ("distant locations"), an intersection changed into a nonintersection, or vice versa. 
change in the response. After the gray screen, the curves appeared again and stayed in view until the subject's response, or when $5 \mathrm{sec}$ had passed since the first appearance of the stimulus. It was not possible for the subject to respond in the curve-tracing task before the gray screen. The subject's response was followed by visual feedback in the tracing task for $1,000 \mathrm{msec}$ (the word correct or wrong). Then the subjects were asked to report, by pressing one of two foot pedals, whether a change in the stimulus had occurred. They were informed that changes could take place for both pairs of curves. We gave no feedback about the accuracy of responses in the change-detection task. After the pedal press, a new trial started. The mappings of the red circle and blue diamond onto the manual responses and the foot pedals onto change-detection responses were counterbalanced across subjects.

We used a within-subjects design and varied three factors: (1) the intersections between the relevant and irrelevant pairs of curves (four levels for both pairs of curves); (2) the SOA between the appearance of the stimulus and the blank screen, which was either short or long; and (3) the change that did or did not occur in the stimulus; if there was a change, it could occur in one of four locations (five levels: no change, plus four types of change trials). The stimulus type (1 out of 128; see above) was counterbalanced across trials, and the other factors were randomized.

As a short SOA, we always chose $100 \mathrm{msec}$. Thus, after the fixation epoch of $1,000 \mathrm{msec}$, the stimulus was in view for $100 \mathrm{msec}$; then the subjects saw a mask of $100 \mathrm{msec}$ followed by the second stimulus. The longer SOA was determined separately for subjects and stimulus types $(\mathrm{OO}, \mathrm{OX}, \mathrm{XO}$, and $\mathrm{XX})$ on the basis of their RT in the baseline block. We chose different SOAs for the different stimulus types because the duration of the tracing process increases with the number of intersections (Houtkamp et al., 2003; Roelfsema et al., 1999). In addition, we determined these values for every subject, because tracing speed differs between subjects. Our aim was to choose an SOA in which attention reaches the distant change location, and we (arbitrarily) chose $66 \%$ of the RT in the baseline block, estimated as the mean RT for the given stimulus type minus $300 \mathrm{msec}$ (as an approximation to the motor component of the response). In a previous study, we used a similar approach and found that the gradual spread of attention can indeed by measured in this way (Houtkamp et al., 2003). The average long SOA across subjects and stimulus types was $582 \mathrm{msec}(414,517,597$, and $802 \mathrm{msec}$ for $\mathrm{OO}, \mathrm{OX}, \mathrm{XO}$, and $\mathrm{XX}$, respectively). Also in this task, the subjects were required to maintain fixation until their response in the curvetracing task. Of all trials, $16 \%$ were aborted due to a fixation error; they were repeated later during the block.

\section{RESULTS}

We will first describe the pattern of RTs in the baseline task where changes did not occur and subjects only traced a curve starting at the fixation point to identify the item at the other end of this curve. We will then present curvetracing performance in the task where the stimulus could change and perceived changes had to be reported at the end of every trial. Finally, we will discuss performance in the change-detection task and compare it with curvetracing performance.

\section{Curve Tracing-Baseline Task}

Once the subjects were accustomed to maintaining fixation, we started the experiment with a baseline block of trials to measure RTs for the different stimuli. Previous studies demonstrated that the RT depends on the number of intersections between the target curve and other curves (Roelfsema et al., 1999), so we expect an effect of the in- tersections between the relevant pair of curves (connected to the fixation point; left in the example of Figure 3) but little effect of the intersections between the irrelevant pair of curves (right in Figure 3). We carried out a two-way ANOVA to determine the effect of intersections between the relevant and irrelevant pairs of curves (OO, OX, XO, and XX in Figure 2) on RT. Thus, the first factor in the ANOVA was the type of stimulus on the relevant side, and the second factor was the stimulus on the irrelevant side. As expected, the stimulus type on the irrelevant side (OO, $\mathrm{OX}, \mathrm{XO}$, and $\mathrm{XX}$ ) did not have a significant effect on the RT $\left[F(3,12)=0.19, M S_{\mathrm{e}}=5.0 \times 10^{4}, p>.5\right]$, whereas there was a clear effect of stimulus type on the relevant side $\left[F(3,12)=29.3, M S_{\mathrm{e}}=4.2 \times 10^{3}, p<.0001\right]$. There was no significant interaction between these factors $\left[F(9,36)=0.72, M S_{\mathrm{e}}=3.1 \times 10^{4}, p>.5\right]$, and we therefore pooled data across configurations of the irrelevant pair to determine the average RT per stimulus type on the relevant side. The RT was $908 \mathrm{msec}$ in the $\mathrm{OO}$ condition, increasing to $1,064 \mathrm{msec}$ and $1,176 \mathrm{msec}$ for the $\mathrm{OX}$ and XO conditions and to $1,495 \mathrm{msec}$ in the XX condition.

\section{Curve Tracing- The Effects of Changes in the Stimulus}

In the main task, the subjects performed the same curvetracing task, but now the stimulus changed on half of the trials. We first investigated whether the increase in RT with the number of intersections also occurred in the task that included changes (Figure 4). In this analysis, we first investigated whether the effect of stimulus type that occurred in the baseline task was also observed in the presence of the stimulus changes. In a first analysis, we therefore averaged across SOAs and also across the close and distant change locations (the next section will examine the effect of SOA and change location). We first compared the accuracy between the conditions and observed an effect of stimulus type $\left[F(1.06,4.25)=11.5, M S_{\mathrm{e}}=1.4 \times 10^{-2}\right.$, $p<.05$, Greenhouse-Geisser correction for nonsphericity], because there were more errors if there were more intersections (bars in Figure 4). In addition, we observed an effect of change condition $\left[F(1.01,4.05)=45.6, M S_{\mathrm{e}}=\right.$ $1.4 \times 10^{-3}, p<.01$, Greenhouse-Geisser correction for nonsphericity], because most errors occurred for changes in the configuration of the relevant curves. The interaction between change condition and stimulus type was also significant $\left[F(6,24)=4.22, M S_{\mathrm{e}}=2.5 \times 10^{-3}, p<.01\right]$.

We next investigated the pattern of RTs. A two-way ANOVA with change condition and stimulus type as factors confirmed that there was a significant effect of stimulus type on the RT $\left[F(3,12)=62.2, M S_{\mathrm{e}}=1.7 \times 10^{4}\right.$, $\left.p<10^{-6}\right]$, in accordance with the results of the baseline task. There was also a main effect of change condition $\left[F(2,8)=43.4, M S_{\mathrm{e}}=1.1 \times 10^{4}, p<.001\right]$. Planned comparisons revealed that RT on trials with a change in the relevant pair of curves was $279 \mathrm{msec}$ longer, on average, than that on no-change trials $\left[F(1,4)=65.4, M S_{\mathrm{e}}=\right.$ $\left.1.1 \times 10^{4}, p<.01\right]$. On the other hand, RTs on trials with a change between the irrelevant curves and no-change trials did not differ significantly $\left[F(1,4)=0.01, M S_{\mathrm{e}}=\right.$ 


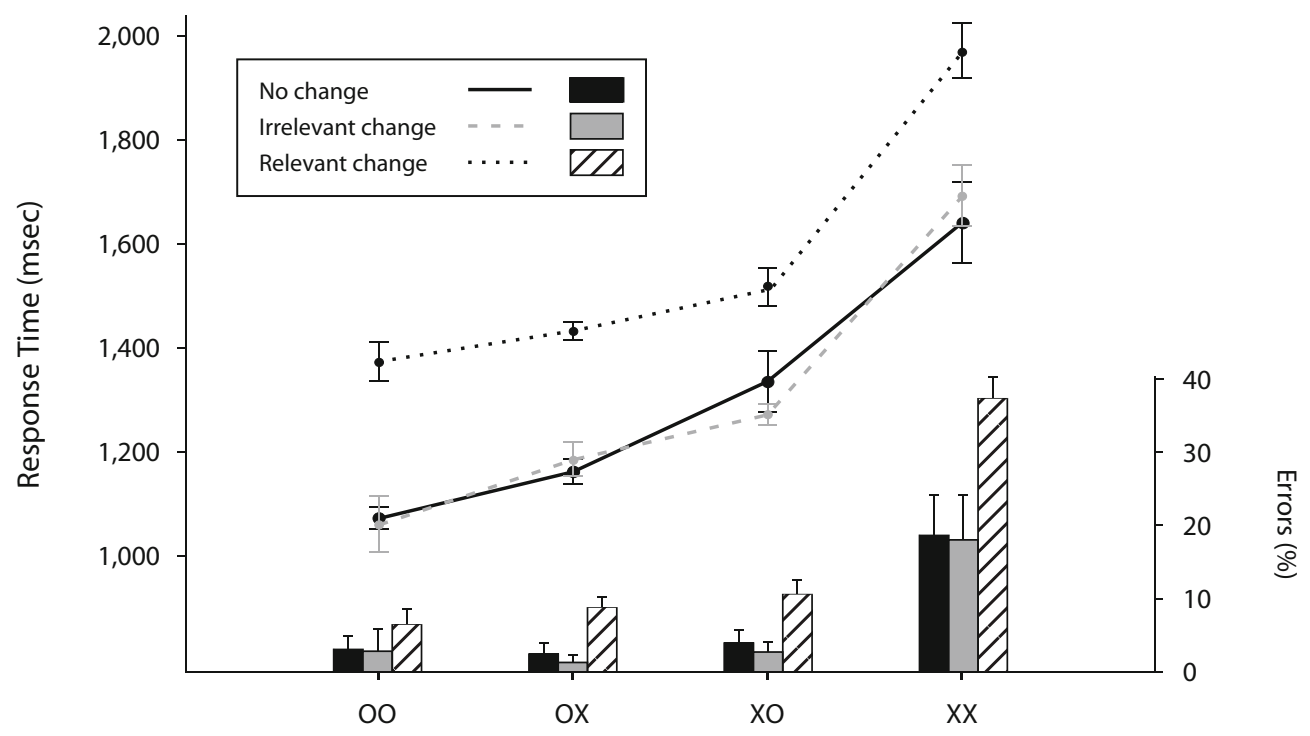

Configuration of the Relevant Curves

Figure 4. Average response times (RTs) and error rates in the primary curve-tracing task. The lines show average RTs for trials without a change (continuous curve), with an irrelevant change (gray dashed curve), and with a relevant change (black dotted) curve. The results are averaged across the short and long stimulus onset asynchronies and across changes at the two possible change locations. Bars show percentage of errors on no-change trials (black bars), irrelevant-change trials (gray bars), and relevant-change trials (striped bars). Error bars indicate SEMs.

$\left.2.4 \times 10^{3}, p>.5\right]$. The interaction between stimulus type and change condition was not significant $[F(6,24)=0.9$, $\left.M S_{\mathrm{e}}=7.4 \times 10^{3}, p>.5\right]$. To increase the power in our additional analyses that aim to elucidate the effect of the different change conditions, we therefore pooled data across different stimulus types (OO, OX, XO, and XX).

\section{Curve Tracing-Effects of Change Location and SOA}

Accuracy. Figure 5A compares the accuracies of the subjects in the curve-tracing task between conditions where the stimulus changed and where it did not, averaged across the different stimulus types. The left and right sets of bars in the figure show the accuracy across subjects for the trials with a short and long SOA, respectively. To investigate whether there were significant differences in accuracy between conditions, we carried out a twoway ANOVA with SOA and change condition as factors. We did not observe a main effect of SOA on accuracy $\left[F(1,4)=6.7, M S_{\mathrm{e}}=3.1 \times 10^{-3}, p>.05\right]$, but there was a significant effect of change condition $[F(4,16)=17.9$, $\left.M S_{\mathrm{e}}=1.8 \times 10^{-3}, p<10^{-5}\right]$. It can be seen in Figure 5A that differences between change conditions occurred only at the long SOA, not at the short SOA. Accordingly, we obtained a significant interaction between these factors $\left[F(4,16)=3.9, M S_{\mathrm{e}}=6 \times 10^{-3}, p<.05\right]$. At the short SOA, the subjects were apparently able to detect those changes in the stimulus that required a change of the response in the curve-tracing task. We note, however, that the high accuracy conveys limited information about the distribution of attention. It is compatible with the possibility that the subjects noticed the change and adjusted their response, but it is also possible that the change location was not yet reached by the tracing process. Changes in contours that are yet to be processed do not cause interference in either model of contour grouping (Figures 1B and $1 \mathrm{C}$ ).

There were significant differences in accuracy between the change conditions at the long SOA. The accuracy on trials without a change was $93 \%$; this was comparable with the accuracy on trials with change on the irrelevant side $(96 \%$ for a close and $96 \%$ for a distant change). Planned comparisons revealed that the accuracy decreased significantly for trials with a change in the relevant pair of curves. Accuracy for trials with a change at the close location was $74 \%$, and this was significantly lower than performance on no-change trials $\left[F(1,4)=14.1, M S_{\mathrm{e}}=6.7 \times 10^{-3}, p<.05\right]$. We obtained a similar result for the trials with a change at the distant location where performance was reduced to $79 \%$, significantly lower than the performance on the no-change trials $\left[F(1,4)=13.9, M S_{\mathrm{e}}=3.7 \times 10^{-3}, p<.05\right]$. The accuracies on trials with a change at the close and distant relevant location did not differ significantly $[F(1,4)=$ $\left.3.1, M S_{\mathrm{e}}=2.1 \times 10^{-3}, p>.15\right]$.

These results agree with those in Crundall et al. (2008), who also found that accuracies in the curve-tracing task decreased at longer SOAs, although the present interaction between SOA and change location was not observed in that study. Crundall et al. concluded from these results 

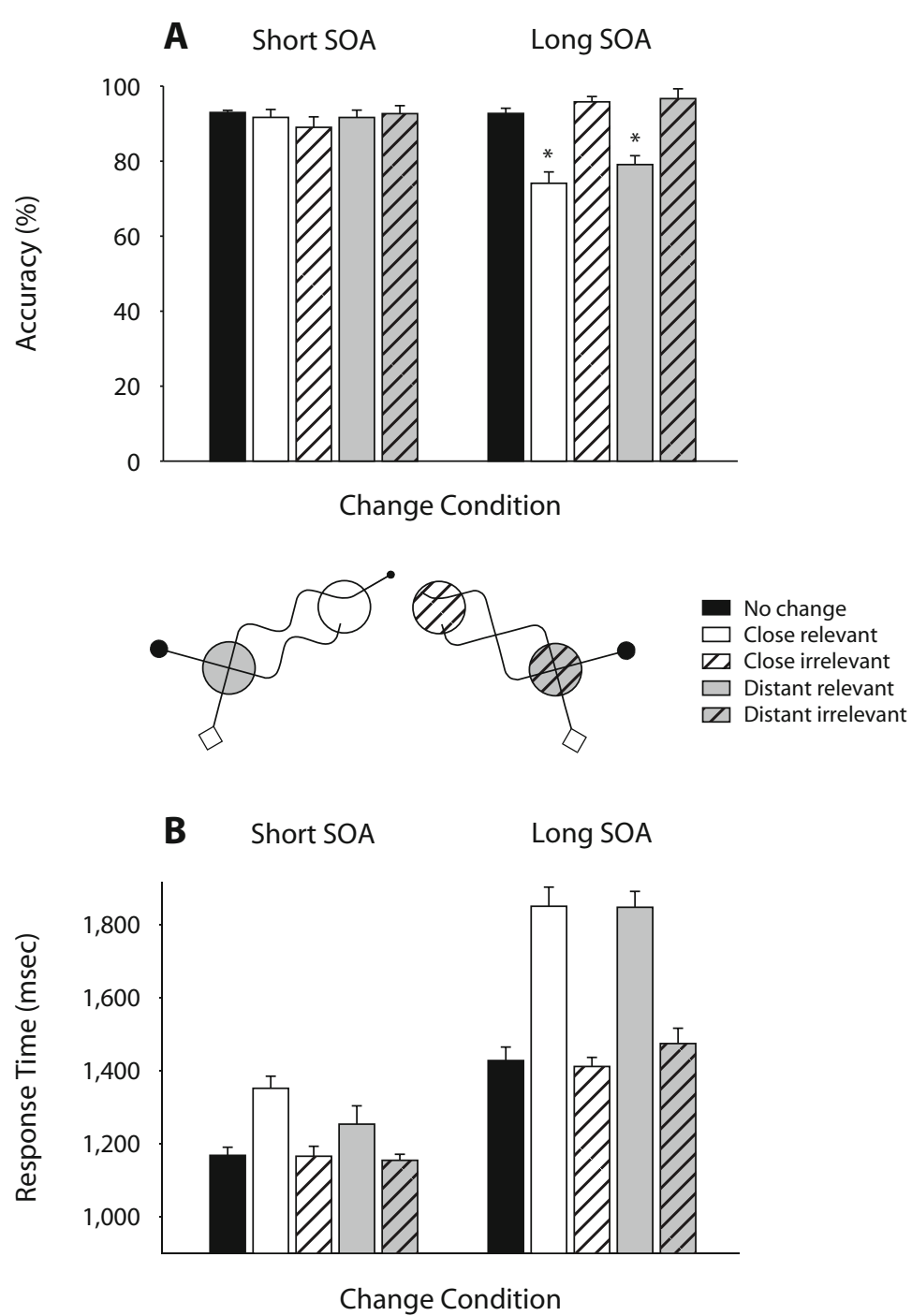

Figure 5. Performance in the curve-tracing task pooled across stimulus types. (A) Accuracy on trials without a change (black bars), trials with a change at the close relevant or irrelevant location (white and white striped bars), and trials with a change at the distant relevant or irrelevant location (gray and gray striped bars). The left five bars show accuracies on trials with a short stimulus onset asynchrony (SOA), and the right bars accuracy on trials with a long SOA. The inset illustrates the various change locations. (B) Response times on correct trials.

that attention had retracted from the initial contour elements of the target curve at the longer SOA, and argued that this result supported the moving zoom lens model. We note, however, that it is possible to predict the performance of a subject who does not perceive any of the changes from the performance on no-change trials. All responses that would be correct on no-change trials change into errors, which would lead to an accuracy of only $7 \%$, much lower than the observed accuracy of $74 \%$. We therefore have to conclude that, even at the long SOA, the majority of changes at the close location were detected, a result compatible with the spreading-attention model.

RTs. Figure 5B shows the RTs in the curve-tracing task. To evaluate the data, we carried out a two-way
ANOVA with factors of SOA and change condition. We obtained a main effect of SOA on the RT $[F(1,4)=224$, $\left.M S_{\mathrm{e}}=8.1 \times 10^{3}, p<.001\right]$, since RTs at the long SOA (1,603 msec on average) were $382 \mathrm{msec}$ longer than RTs at the short SOA $(1,221 \mathrm{msec})$. The increase in RT with SOA even occurred on the no-change trials, in which RTs increased by $259 \mathrm{msec}$, from $1,171 \mathrm{msec}$ at the short SOA to $1,430 \mathrm{msec}$ at the long SOA. This effect of SOA is in accordance with the results in Crundall et al. (2008), and indicates that the gray mask presented for $100 \mathrm{msec}$ interfered with the contour-grouping process, even if the stimulus remained constant. In addition, we obtained a main effect of change condition $\left[F(4,16)=34.6, M S_{\mathrm{e}}=\right.$ $6.8 \times 10^{3}, p<10^{-6}$ ]. Planned comparisons revealed that 
the RTs on trials with a change at a relevant location were significantly longer ( $279 \mathrm{msec}$ on average) than those on trials without a change $\left[F(1,4)=65.4, M S_{\mathrm{e}}=7.9 \times 10^{4}\right.$, $p<.01]$. RTs on irrelevant-change trials were not significantly longer than those on no-change trials $[F(1,4)=$ $\left.0.01, M S_{\mathrm{e}}=1.6 \times 10^{3}, p>.5\right]$.

Finally, we observed a significant interaction between SOA and change condition $\left[F(4,16)=14.6, M S_{\mathrm{e}}=\right.$ $4.0 \times 10^{3}, p<10^{-4}$ ], reflecting a larger influence of SOA on change trials. This effect can be explained by the requirement to reprogram the manual response, a process that presumably takes longer toward the end of a trial. It is also possible, however, that subjects had to retrace the relevant curve. More retracing would be required for changes at the longer SOA, resulting in a larger increase in RT.

\section{Change-Detection Performance}

After their response in the curve-tracing task, subjects had to indicate whether they had perceived a change in the stimulus. We evaluated change-detection performance as another measure for the distribution of attention. On nochange trials, the accuracies for reporting the absence of a change were $89 \%$ and $91 \%$ for trials with a short and long SOA, respectively. It follows that the subjects committed a false alarm on $11 \%$ and $9 \%$ of the no-change trials (black bars in Figure 6). Change-detection performance is expected to equal the false alarm rate if subjects fail to notice the changes, and we therefore compared the accuracies with the false alarm rate on no-change trials.

The first main effect observed in the two-way ANOVA was an influence of SOA on change detection. Change detection was better at the longer SOA $[F(1,4)=11.7$, $\left.M S_{\mathrm{e}}=8.7 \times 10^{-3}, p<.05\right]$. In addition, we observed a main effect of change condition $[F(1.68,6.7)=68.5$, $M S_{\mathrm{e}}=1.8 \times 10^{-2}, p<10^{-4}$, Greenhouse-Geisser correction for nonsphericity]. Change detection on the irrelevant side was remarkably poor, since subjects detected only $9 \%$ of the changes at the close as well as at the distant location, and a planned comparison revealed that these accuracies did not differ significantly from the false alarm rate $\left[F(1,4)=0.34, M S_{\mathrm{e}}=1.2 \times 10^{-3}, p>.5\right]$. Change detection for the relevant pair of curves was much better and differed from the irrelevant-change trials at both change locations for the short [close location, $F(1,4)=$ $37.5, M S_{\mathrm{e}}=2.3 \times 10^{-2}, p<.01$; distant location, $\left.F(1,4)=29.2, M S_{\text {e }}=1.4 \times 10^{-2}, p<.01\right]$ and the long SOA [close location, $F(1,4)=86.7, M S_{\mathrm{e}}=1.8 \times 10^{-2}$, $p<.001$; distant location $F(1,4)=190, M S_{\mathrm{e}}=8.1 \times$ $\left.10^{-3}, p<.001\right]$. Critically, the change-detection accuracy on relevant-change trials showed a pattern opposite that for accuracy in the curve-tracing task: Performance in the curve-tracing task decreased at the long SOA if the stimulus changed, whereas the detection of these changes improved. On the premise that change-detection performance provides a measure for the distribution of visual attention, our results imply that the subjects paid attention to both locations at the long SOA, which is incompatible with a moving zoom lens of attention.
Short SOA

Long SOA

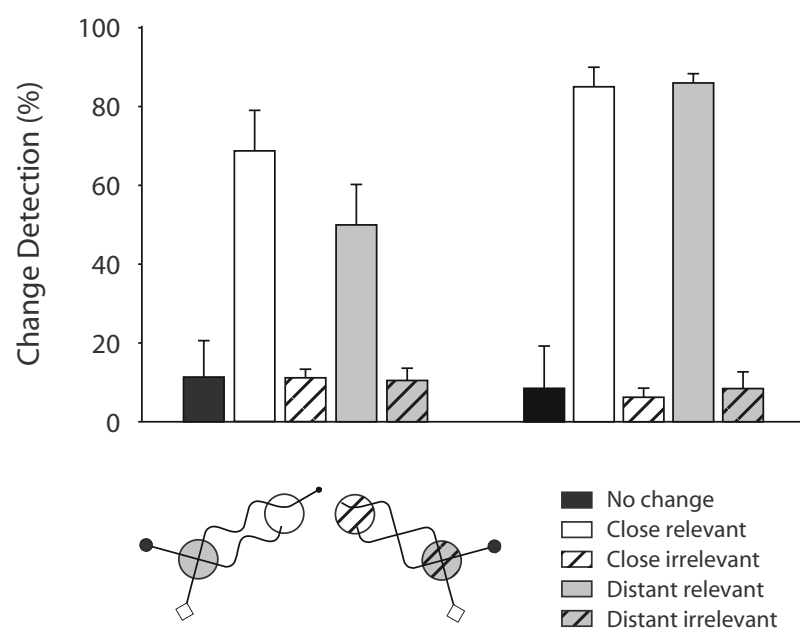

Figure 6. Change-detection accuracies. Bars illustrate the percentage of trials where a change was reported by the subjects. Black bars show the false alarm rate on trials without a change. White or white striped bars show change-detection accuracy on trials with a close relevant or irrelevant change, and gray or gray striped bars show accuracy on trials with a distant relevant or irrelevant change, respectively. Left bars show data from the trials with a short stimulus onset asynchrony (SOA), and the right bars data from trials with a long SOA.

\section{Comparison Between Curve-Tracing and Change-Detection Performance}

It is of interest to compare the accuracy in the curvetracing task to the change-detection performance. We reproduced the finding of Crundall et al. (2008) that curve-tracing accuracy decreased at the longer SOA if the stimulus changed and found, in addition, that this effect was most pronounced at the close location where the accuracy decreased to $74 \%$. Does this imply that the changes were missed on $26 \%$ of trials, and that attention had retracted on these trials from the initial contour elements of the target curve?

The change-detection accuracies suggest not. Subjects detected the changes at the close location at the long SOA with an accuracy of $86 \%$, but they apparently failed to use this information to correct the curve-tracing response on a fraction of the trials. To further investigate the discrepancy between the two measures of change detection, we selected only the trials with relevant changes at the close location at the long SOA that were detected by the subjects. Although the subjects were aware of the change on these trials, they reached an accuracy of only $77 \%$ in the curve-tracing task (slightly better than the accuracy of $74 \%$ across all trials, with a late change at the close relevant location), and a one-way ANOVA indicated that this was significantly lower than the accuracy of $93 \%$ on the no-change trials $\left[F(1,4)=8.01, M S_{\mathrm{e}}=7.5 \times 10^{-3}\right.$, $p<.05]$. Conversely, on the trials with a late change at the close location that caused an error in the curve-tracing task, subjects were able to report the change on $70 \%$ of the trials, and a one-way ANOVA revealed that this is sig- 
nificantly better than the false alarm rate of $8 \%$ on correct trials $\left[F(1,4)=19.9, M S_{\mathrm{e}}=6.7 \times 10^{-2}, p<.05\right]$. Apparently, the subjects failed to modify their response on a fraction of the trials, although they detected the change. The important implication of this finding is that the performance in the curve-tracing task on change trials does not accurately measure change-detection performance.

\section{DISCUSSION}

Here, we have investigated a paradigm introduced by Crundall et al. (2008) that combines a contour-grouping task with a change-detection task to measure the distribution of attention. The logic behind the task is that subjects detect changes only in attended regions of a stimulus, so that the accuracy on the change trials can be used to measure the distribution of attention. We reproduced the main findings of the study by Crundall et al., because we found that (1) accuracy in the curve-tracing task decreases if the change occurs at a long SOA, (2) RT increases if the mask occurs at a later point in time, and (3) RT is longer on change trials than on no-change trials. Furthermore, we asked the subjects to report any changes that they perceived. The additional results show that lower curvetracing performance is not accompanied by a failure to detect changes in the stimulus and thereby call the usefulness of the paradigm into question.

\section{Evaluation of the Combined Contour-Grouping and Change-Detection Paradigms}

One of the proclaimed advantages of the combined contour-grouping change-detection task is that it permits the measurement of the distribution of attention without the use of a secondary task, unlike the studies by Scholte et al. (2001) and Houtkamp et al. (2003), who combined a curve-tracing task with a color-report task. Although we agree that secondary tasks may influence performance, the studies by Scholte et al. and Houtkamp et al. took a number of measures to discourage changes in strategy, and thereby ensured that the pattern of RTs was not changed by the secondary task. In contrast, the results of the present experiment and the study of Crundall et al. (2008) provide clear indications that the inclusion of change trials influences the subjects' strategy. This should come as no surprise, because Crundall et al. changed the stimulus on $50 \%$ of the trials, causing the stimulus before the mask to be uncorrelated with the required response. A strategic subject might therefore disregard the stimulus before the mask. The present study partially alleviated this problem by also including trials with a change in an irrelevant pair of curves, so that an inversion of the response was required on only $25 \%$ of trials. Nevertheless, we obtained clear evidence for changes in strategy. First, the RTs increased on trials with a long SOA (in accordance with Crundall et al., 2008). This suggests that subjects reserved additional time to take into account potential changes in the stimulus. Second, we replicated the finding of Crundall et al., that is, that the subjects are slower on change trials than on nochange trials. This finding indicates that attention does not spread or move in an undisturbed manner after a change in the stimulus. Third, we observed a significant interaction between SOA and change condition (Figure 5), providing additional evidence that the changes in the stimulus interfered with the contour-grouping process. These results, taken together, indicate that a flashing full-screen mask, followed by a change in the stimulus on a substantial fraction of trials, has an influence on the deployment of attention. Thus, the process responsible for contour grouping changes in the presence of change trials.

Another finding that casts doubt on the usefulness of the new paradigm is the discrepancy between the curvetracing performance and change detection on the change trials. The performance in the contour-grouping task on change trials measures attention accurately neither on trials with a short SOA nor on trials with a long SOA. At the short SOA, curve-tracing performance was high for both relevant change locations. The good performance, however, does not reveal whether the change location is not yet reached by the tracing process, or whether attention has arrived and changes are detected. At the long SOA, another problem emerged. We found that the subjects did not always compensate for the changes they detected, and performance in the curve-tracing task therefore underestimated change-detection accuracy.

There are at least two possible explanations for the failure of subjects to modify their response if they detected the change. First, it is conceivable that the change occurred too late for a modification of a prepared response. However, in their Experiment 3, Crundall et al. (2008) provided some evidence against this possibility. They revealed the identity of the items at the end of the curves (in our experiment, the diamond or circle; see Figure 3) after the mask, so that subjects could not prepare their response before the change in the stimulus. In this experiment, the accuracy of the subjects was also reduced on change trials with a long SOA, indicating that the failure to cancel a planned response cannot be the only explanation for the decrease in performance.

Second, the subjects may have to retrace the target curve if they detect the change. Such a retracing process can explain why the RTs on trials with a relevant change were longer than those on the irrelevant-change and no-change trials, where retracing was unnecessary. Furthermore, retracing should cause the largest increase in RTs on change trials with a long SOA, because the subject has to retrace a longer segment of the target curve; this is precisely what we and Crundall et al. (2008) observed. A retracing process would also account for the discrepancy between the change-detection performance and the curve-tracing accuracy on change trials. Here, we found that curve-tracing performance was slightly (although not significantly) better with distant than with close changes on trials with a long SOA, although change-detection accuracy was the same for both locations. Subjects may not have compensated for all the changes in the stimulus that they detected, especially if the detected change was close to the fixation point, and they therefore may have responded on some of these trials before retracing the entire target curve. 


\section{Implications for Models of Contour Grouping}

Crundall et al. (2008) concluded that the decrease in performance on trials with a change at the start of the target curve at the longer SOA implies that attention retracts from the initial segments of the curve, which would be evidence in favor of the moving zoom lens model (Figure 1C). The present results cast doubt on this conclusion, because the decrease in performance at the start of the target curve was not reproduced with an explicit change-detection task. Instead, change detection was equally good for close and distant changes in the relevant pair at the long SOA. In contrast, the change-detection performance for the irrelevant pair was at chance level, which implies that change detection was not possible without attention. The important implication is that the good change-detection performance for the relevant curves indicates that these curves were attended. The overall pattern of change-detection results (Figure 6) therefore indicates that attention was eventually directed to the close and distant contour elements of the relevant curve, which were attended at the same time. These results support the spreading-attention model, and are incompatible with the moving zoom lens model.

The present results are in accordance with previous studies that combined a curve-tracing task with a colorreport task (Houtkamp et al., 2003; Scholte et al., 2001). In these studies, subjects had to remember colors on the target or on the distractor curve; we observed that color reports were initially better at the start of the target curve, and that the superior performance then gradually spread across all the contour elements of the target curve. Thus, the present and previous results, taken together, imply that attention spreads across the target curve, so that the contour elements to be grouped in perception are all labeled by object-based attention (Figure 1B).

We considered the possibility that the additional question about perceived changes at the end of every trial may have had an influence on the distribution of attention, over and above the interference caused by the changes in the stimulus. On trials with a relevant change, it was essential for the subjects to register this change for a correct response in the curve-tracing task, and it therefore seems unlikely that questions about these relevant changes changed the deployment of attention. We were more concerned with the possibility that the questions could also cause subjects to direct attention to the irrelevant side. Fortunately, this possibility was ruled out by the remarkable inability of the subjects to notice changes on the irrelevant side. Thus, it seems unlikely that the additional question influenced the deployment of attention.

\section{The Neurophysiology of Contour Grouping}

Studies that assess the spatial distribution of attention by the measurement of brain activity need not be concerned with the possibility of changes in the subjects' strategy. In a series of neurophysiological experiments, we found that the neuronal activity evoked by contour elements of a target curve in the visual cortex of macaque monkeys is enhanced over the activity evoked by distracting curves (Roelfsema et al., 1998; Roelfsema \& Spekreijse, 2001). These results imply that contours to be grouped in percep- tion are labeled in the visual cortex with an enhanced neuronal response (Roelfsema, 2006). This selective labeling process occurs even if the target curve crosses a distracting curve, because neurons coding contours of the target curve simultaneously enhance their response, even if these contours are on opposite sides of the intersection. These results support the involvement of object-based attention, and are incompatible with an attentional zoom lens.

The attentional enhancement of neuronal activity does not occur during an initial, preattentive processing phase that starts $40 \mathrm{msec}$ after the presentation of the stimulus, but rather after a delay of 140-200 msec (Roelfsema, Khayat, \& Spekreijse, 2003; Roelfsema, Tolboom, \& Khayat, 2007). We recently investigated the delay between the initial visual response and the enhancement of the response and found that it depends on the distance between the neuron's receptive field and the start of the tracing process (Pooresmaeili \& Roelfsema, 2010). Neurons with receptive fields close to the start of the target curve enhance their response at an earlier point in time than do neurons with a receptive field further along the curve. Moreover, neurons coding contour elements of the start of the curve maintain their elevated response, whereas the response enhancement is propagated to the representation of other contour elements. These results, taken together, imply a remarkable correspondence between the neurophysiology of perceptual grouping and the spread of attention model (Figure 1B).

\section{Incremental Grouping \\ by the Spread of Attention}

In the introduction, we outlined two mechanisms for perceptual grouping. We hypothesized a fast mechanism for the coding of perceptual groups by dedicated neurons in higher areas that are tuned to, for example, elongated contours of a particular shape (Brincat \& Connor, 2006). These neurons are activated shortly after the presentation of the visual stimulus and in parallel across the visual scene. We called these hardwired groupings "base groupings" and suggested that they are detected automatically in preattentive vision (Roelfsema, 2006).

It is unlikely, however, that there are neurons tuned to new contours of any arbitrary shape like the shape of the cables in Figure 1A; and yet we do not experience difficulties when we see a curve with a shape that we never saw before. In these situations, we exploit the Gestalt grouping rules of connectedness and good continuation (Kellman \& Shipley, 1991) that establish relationships between nearby contour elements of the same curve. Contour elements that are farther apart are linked only indirectly by a chain of groupings, which, we propose, are formed by objectbased attention that propagates along the local groupings until the entire curve is labeled by attention. The flexibility of this "incremental grouping" process comes at the cost of a longer processing time, which increases linearly with the length of the target curve (Jolicœur et al., 1991) and also with the number of crossings with other, distracting curves (Roelfsema et al., 1999).

It is important to emphasize that we do not propose that complex and elongated curves are always entirely 
labeled by attention. It is likely that there is an upper limit on the number of image elements that can be grouped incrementally, although the present results, the findings of Houtkamp et al. (2003), and the neurophysiological studies demonstrate that this limit is larger than the segment of a curve that fits into a zoom lens. If a curve is very contorted, object-based attention might retract from the initial segments but spread over image elements farther along the curve. Such a limit would be in line with other work demonstrating that only a few image elements can be attended at the same time. For example, Pylyshyn and Storm (1988) observed an upper limit of about four in a task in which subjects had to track independently moving image elements. Yantis (1992) suggested that subjects perform this tracking task by grouping the elements into a dynamically morphing object. Future work could investigate whether similar limitations occur in contour-grouping tasks, where object-based attention binds contour elements incrementally to form coherent object representations.

\section{Relation to Previous Studies on Object-Based Attention}

Incremental grouping requires that attention spreads from image elements that are attended to other elements linked by Gestalt grouping cues. This spread of attention through local groupings is supported by studies using a large variety of techniques, including cuing tasks (Egly et al., 1994; Goldsmith \& Yeari, 2003; Lamy \& Egeth, 2002; Shomstein \& Behrmann, 2008; Vecera, 1994), divided-attention tasks (Behrmann, Zemel, \& Mozer, 1998; Watson \& Kramer, 1999), flanker tasks (Baylis \& Driver, 1992; Driver \& Baylis, 1989; Kramer \& Jacobson, 1991; Richard et al., 2008), and visual search (Banks \& Prinzmetal, 1976; Donnelly, Humphreys, \& Riddoch, 1991; Duncan \& Humphreys, 1989; Wolfe \& Bennett, 1997). A topic of ongoing debate is whether attention automatically spreads across image elements that form a perceptual group (Shomstein \& Yantis, 2002). The flanker task is a good test for the automatic spread of attention, because it is to the subject's advantage to focus on the central item and not to attend to the flankers. An influence of grouping on flanker interference therefore suggests that subjects have little control over the spread of attention from the target to the flankers. Recent studies demonstrated that there are conditions favoring such an automatic spread of attention. Richard et al. (2008), for example, demonstrated that attention spreads automatically if the to-be-reported features are integral parts of the central object and the flankers. Chen and Cave (2006) observed an automatic spread of attention if the grouping of the central target with the flankers is made strong enough. Object-based effects are also pronounced in tasks demanding attention shifts, which are more efficient between locations on the same object than between locations on different objects (Lamy \& Egeth, 2002).

We note that many of these approaches to studying object-based attention are indirect, because they investigate the side effect of grouping on performance in another task. In contrast, the present contour-grouping task directly tests the ability of subjects to report whether contour elements do or do not belong to the same curve. Here, the subject's task is to build larger perceptual groups by combining local groupings incrementally. The present and previous studies, taken together, indicate that the spread of attention is the key mechanism for the incremental construction of coherent object representations. Whether the object-based effects that occur in cuing tasks, flanker tasks, and visual search are side effects of the ability to build incremental groups, or whether there are additional benefits of the spread of attention for perception, promises to be an exciting avenue for future research.

\section{AUTHOR NOTE}

This work was supported by an HFSP Investigators Grant, a grant from the European Union (EU IST Cognitive Systems, Project 027198 "Decisions in Motion"), a grant of NWO Exact, and an NWO VICI grant awarded to P.R.R. Correspondence concerning this article should be addressed to P. R. Roelfsema, Netherlands Institute for Neuroscience, Meibergdreff 47, 1105 BA Amsterdam, The Netherlands (e-mail: p.roelfsema@nin.knaw.nl).

\section{REFERENCES}

Avrahami, J. (1999). Objects of attention, objects of perception. Perception \& Psychophysics, 61, 1604-1612.

BanKs, W. P., \& Prinzmetal, W. (1976). Configurational effects in visual information processing. Perception \& Psychophysics, 19, 361-367.

BAYLIS, G. C., \& Driver, J. (1992). Visual parsing and response competition: The effect of grouping factors. Perception \& Psychophysics, 51, 145-162.

Behrmann, M., Zemel, R. S., \& Mozer, M. C. (1998). Object-based attention and occlusion: Evidence from normal participants and a computational model. Journal of Experimental Psychology: Human Perception \& Performance, 24, 1011-1036.

Brincat, S. L., \& Connor, C. E. (2006). Dynamic shape synthesis in posterior inferotemporal cortex. Neuron, 49, 17-24.

Chen, Z., \& Cave, K. R. (2006). Reinstating object-based attention under positional certainty: The importance of subjective parsing. Perception \& Psychophysics, 68, 992-1003.

Crundall, D., Dewhurst, R., \& Underwood, G. (2008). Does attention move or spread during mental curve tracing? Perception \& Psychophysics, 70, 374-388.

Donnelly, N., Humphreys, G. W., \& Riddoch, M. J. (1991). Parallel computation of primitive shape descriptions. Journal of Experimental Psychology: Human Perception \& Performance, 17, 561-570.

Driver, J., \& BAYLIS, G. C. (1989). Movement and visual attention: The spotlight metaphor breaks down. Journal of Experimental Psychology: Human Perception \& Performance, 15, 448-456.

DunCAN, J. (1984). Selective attention and the organization of visual information. Journal of Experimental Psychology: General, 113, 501-517.

Duncan, J., \& HumphreYs, G. W. (1989). Visual search and stimulus similarity. Psychological Review, 96, 433-458.

Egly, R., Driver, J., \& Rafal, R. D. (1994). Shifting visual attention between objects and locations: Evidence from normal and parietal lesion subjects. Journal of Experimental Psychology: General, 123, 161-177.

ERIKSEN, B. A., \& ERIKSEN, C. W. (1974). Effects of noise letters upon the identification of a target letter in a nonsearch task. Perception \& Psychophysics, 16, 143-149.

Goldsmith, M., \& Yeari, M. (2003). Modulation of object-based attention by spatial focus under endogenous and exogenous orienting. Journal of Experimental Psychology: Human Perception \& Performance, 29, 897-918.

HoutKamp, R., Spekreijse, H., \& Roelfsema, P. R. (2003). A gradual spread of attention during mental curve tracing. Perception \& Psychophysics, 65, 1136-1144.

Hung, C. P., Kreiman, G., Poggio, T., \& DiCarlo, J. J. (2005). Fast readout of object identity from macaque inferior temporal cortex. Science, 310, 863-866. 
Joliceleur, P., \& InGLETON, M. (1991). Size invariance in curve tracing. Memory \& Cognition, 19, 21-36.

Jolicceur, P., Ullman, S., \& Mackay, M. (1986). Curve tracing: A possible basic operation in the perception of spatial relations. Memory \& Cognition, 14, 129-140.

Joliceur, P., Ullman, S., \& Mackay, M. (1991). Visual curve tracing properties. Journal of Experimental Psychology: Human Perception \& Performance, 17, 997-1022.

Kellman, P. J., \& Shipley, T. F. (1991). A theory of visual interpolation in object perception. Cognitive Psychology, 23, 141-221.

Kramer, A. F., \& Jacobson, A. (1991). Perceptual organization and focused attention: The role of objects and proximity in visual processing. Perception \& Psychophysics, 50, 267-284.

Lamy, D., \& EgETH, H. (2002). Object-based selection: The role of attentional shifts. Perception \& Psychophysics, 64, 52-66.

McCormick, P. A., \& Joliceur, P. (1994). Manipulating the shape of distance effects in visual curve tracing: Further evidence for the zoom lens model. Canadian Journal of Experimental Psychology, 48, 1-24.

Oram, M. W., \& Perrett, D. I. (1992). Time course of neural responses discriminating different views of the face and head. Journal of Neurophysiology, 68, 70-84.

Oram, M. W., \& Perrett, D. I. (1994). Modeling visual recognition from neurobiological constraints. Neural Networks, 7, 945-972.

Pooresmaeili, A., \& Roelfsema, P. R. (2010). Neuronal activity in area V1 during contour grouping conforms to a growth cone model of object-based attention. Manuscript in preparation.

PyLYshyn, Z. W., \& StoRM, R. W. (1988). Tracking multiple independent targets: Evidence for a parallel tracking mechanism. Spatial Vision, 3, 179-197.

ReYNolds, J. H., \& Chelazzi, L. (2004). Attentional modulation of visual processing. Annual Review of Neuroscience, 27, 611-647.

Richard, A. M., LeE, H., \& Vecera, S. P. (2008). Attentional spreading in object-based attention. Journal of Experimental Psychology: Human Perception \& Performance, 34, 842-853.

Riesenhuber, M., \& Poggio, T. (1999). Are cortical models really bound by the "binding problem"? Neuron, 24, 87-93.

Roelfsema, P. R. (2006). Cortical algorithms for perceptual grouping. Annual Review of Neuroscience, 29, 203-227.

Roelfsema, P. R., Khayat, P. S., \& Spekreijse, H. (2003). Subtask sequencing in the primary visual cortex. Proceedings of the National Academy of Sciences, 100, 5467-5472.

Roelfsema, P. R., Lamme, V. A. F., \& Spekreijse, H. (1998). Objectbased attention in the primary visual cortex of the macaque monkey. Nature, 395, 376-381.

Roelfsema, P. R., Scholte, H. S., \& Spekreijse, H. (1999). Temporal constraints on the grouping of contour segments into spatially extended objects. Vision Research, 39, 1509-1529.
Roelfsema, P. R., \& Spekreisse, H. (2001). The representation of erroneously perceived stimuli in the primary visual cortex. Neuron, $\mathbf{3 1}$, 853-863.

Roelfsema, P. R., Tolboom, M., \& Khayat, P. S. (2007). Different processing phases for features, figures, and selective attention in the primary visual cortex. Neuron, 56, 785-792.

Rousselet, G. A., Macé, M. J.-M., \& Fabre-Thorpe, M. (2003). Is it an animal? Is it a human face? Fast processing in upright and inverted natural scenes. Journal of Vision, 3, 440-455.

Scholte, H. S., Spekreisse, H., \& Roelfsema, P. R. (2001). The spatial profile of visual attention in mental curve tracing. Vision Research, 41, 2569-2580.

Shomstein, S., \& Behrmann, M. (2008). Object-based attention: Strength of object representation and attentional guidance. Perception \& Psychophysics, 70, 132-144.

Shomstein, S., \& YANTIS, S. (2002). Object-based attention: Sensory modulation or priority setting? Perception \& Psychophysics, 64, 41-51.

Sugase, Y., Yamane, S., Ueno, S., \& Kawano, K. (1999). Global and fine information coded by single neurons in the temporal visual cortex. Nature, 400, 869-872.

TANAKA, K. (1993). Neuronal mechanisms of object recognition. Science, 262, 685-688.

Thorpe, S., Fize, D., \& Marlot, C. (1996). Speed of processing in the human visual system. Nature, 381, 520-522.

Tsao, D. Y., Freiwald, W. A., Tootell, R. B. H., \& Livingstone, M. S. (2006). A cortical region consisting entirely of face-selective cells. Science, 311, 670-674.

VECERA, S. P. (1994). Grouped locations and object-based attention: Comment on Egly, Driver, \& Rafal (1994). Journal of Experimental Psychology: General, 123, 316-320.

Watson, S. E., \& Kramer, A. F. (1999). Object-based visual selective attention and perceptual organization. Perception \& Psychophysics, 61, 31-49.

Wolfe, J. M., \& Bennett, S. C. (1997). Preattentive object files: Shapeless bundles of basic features. Vision Research, 37, 25-43.

Wong, E., \& Weisstein, N. (1983). Sharp targets are detected better against a figure, and blurred targets are detected better against a background. Journal of Experimental Psychology: Human Perception \& Performance, 9, 194-202.

YANTIS, S. (1992). Multielement visual tracking: Attention and perceptual organization. Cognitive Psychology, 24, 295-340.

YeshuRUn, Y., \& CARRASCO, M. (1998). Attention improves or impairs visual performance by enhancing spatial resolution. Nature, 396, 72-75.

(Manuscript received July 9, 2009;

revision accepted for publication November 4, 2009.) 